\title{
Ebolavirus: pseudotypes, libraries and standards
}
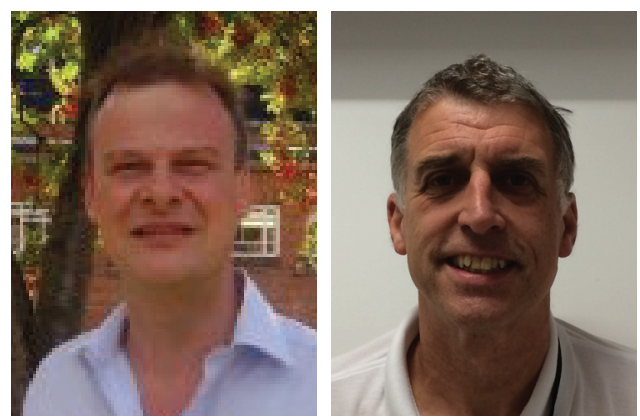

Nigel J Temperton ${ }^{*, 1} \&$ Mark Page $^{2}$
“In order to be able to respond in a

timely and effective manner to

future filovirus outbreaks, a library

of pseudotyped vectors bearing

the GP of filoviruses of major public

and animal health importance

should be prioritized...”
Globalization, accompanied by increasing levels of international travel and trade, climate change, altered human behavior and demographics, is leading to the emergence of manifold viral diseases, many of which are highly pathogenic and hence are considered of great public and animal health importance to the global populace [1]. An excellent, if devastating, example of this is represented by the filovirus family, to which Ebolavirus and Marburgvirus belong. To undertake basic virological research and novel therapeutic development, filoviruses require expert handling and manipulation by highly trained staff within expensive CL-4 facilities not readily available to the virology research community [2]. In order to circumvent the enhanced biosafety requirement, the development of nonpathogenic, replication-defective pseudotyped vectors, developed historically for gene therapy applications, is an effective and established solution to permit the study of many facets of filovirus biology and virus therapeutics in a low containment CL-1/2 laboratory [3]. Ebola virus glycoprotein (GP) pseudotypes constructed with murine leukemia virus cores were first described in 1998 and have been used since then in a broad range of studies [4].

In order to be able to respond in a timely and effective manner to future filovirus outbreaks, a library of pseudotyped vectors bearing the GP of filoviruses of major public and animal health importance should be prioritized. These pseudotype viruses can be readily used for sero-surveillance, $\mathrm{mAb} / \mathrm{antiviral}$ screening, vaccine immunogenicity studies and for tropism/pathogenicity studies as soon as the nucleotide sequence of the GP is online. Such a collection has been instigated by Edward Wright's group at the University of Westminster and includes to date, all five Ebolavirus species including the Makona variant (Guinea, 2014) implicated in the current outbreak [5]. These pseudotypes are currently being utilized as surrogate antigens in virus neutralization assays as part of immunogenicity studies of the vaccine ChAd3 EBOZ at the Jenner Institute (University of Oxford, UK) [6]. Pseudotypes of Zaire Ebolavirus (Mayinga), Bundibugyo Ebolavirus (2008 isolate) and Marburg virus (Lake Victoria

\section{KEYWORDS}

- emerging pathogens $\bullet$ pandemic preparedness $\bullet$ surveillance $\bullet$ viral pseudotypes

“...pseudotypes are currently being utilized as surrogate antigens in virus neutralization assays as part of immunogenicity studies...” 
"Pseudotype viruses and lentiviral vectors have been particularly useful in producing reference standards for Ebola in a relatively quick timeframe...” isolate) were recently employed to screen repurposed drugs that inhibit virus entry [7].

In order to identify and prioritize candidate filovirus GPs for pseudotyping onto retroviral (and other) vector cores, and incorporation of these into the proposed library resource, the following sources (among others) could be employed: WHO/OiE/ProMed/MSF/CDC news channels/feeds, submitted conference abstracts, stakeholder-transmitted information via email, web forums, Twitter, preprints (bioRxiv, PeerJ PrePrints), postpublication review journals (F1000Research), traditional peer-reviewed open access (OA) literature (human/animal outbreaks/surveillance data/vaccine studies), phylogenetic analysis, antigenic cartography, epitope mapping and structural analysis. Once identified, custom synthesis of filovirus GP from candidate strain nucleic acid sequences in open access databases (NCBI), and subcloning into expression vectors using an array of different promoters (CMV, chicken $\beta$-actin) to effect highlevel expression in retroviral vector producer cells (usually HEK293T) can be undertaken. For many laboratories the use of HIV or murine leukemia virus vector systems for pseudotyping is the gold standard although other options are available (SIV/EIAV/VSV vectors).

The development of Ebolavirus GP pseudotyped vectors carrying different reporter genes (GFP/RFP, firefly/renilla $L u c, B$-gal, $S E A P)$ for translation to end-users in a range of sectors (pharma/high-throughput, low resource/field, multiplex, etc.) is warranted as this significantly expands the utility and impact of the resource globally. Marburg virus pseudotypes can be readily lyophilized and reconstituted representing a new avenue for the development of pseudotype virus-based kits for use in low resource settings [8]. Similar kits should be developed for Ebola and evaluated in the field during the ongoing outbreak if possible.

The hosts of this proposed resource library should also be responsible for optimization of protocols (pseudotype production) and standardization of virus stocks depending on enduser assay requirements and laboratory facilities. They should additionally provide materials for the development of standards as developed in the UK by NIBSC. By using a biological reference material or standard of known activity or potency, bioassay results can be compared and calibrated to give a consistent result, no matter when or where the bioassay is performed. In the case of diagnostic tests, the use of control samples of known activity allows calibration of the limits of detection and quantitation as well as the specificity of the test system to be monitored and quality assured. This information is particularly important in emergency situations where accurate diagnosis is vital leading to appropriate clinical management in health care settings. Further, in vaccine efficacy trials, antibody reference standards help to harmonize assay data between laboratories and to determine the vaccine potency by comparing protective antibody titers identified from preclinical studies in animal models, as shown recently for pandemic influenza H1N1 [9]. For Ebola, it remains to be determined what the correlate of protection is, if it is antibody then what protective antibody level does a vaccine need to induce?

Pseudotype viruses and lentiviral vectors have been particularly useful in producing reference standards for Ebola in a relatively quick timeframe. Nucleic acid test (NAT) standards for PCR assays and antigen standards have been designed using these systems to circumvent the use of inactivated virus preparations which usually comprise NAT/antigen standards for viral diagnostics. Reference materials should exhibit commutability and behave as close as possible to the clinical sample; wild-type viruses/antigens are desirable in this regard [10]. However, preparation of inactivated hazard group 3 and 4 viruses is a protracted process because of the need to carry out the work in a high containment laboratory. There are also issues over the assurance of the inactivation process to laboratories receiving these types of reference materials. Does the inactivation process required (irradiation, chemical treatments) destroy the nucleic acid and/or denature the antigenicity of the protein? Pseudotype viruses and vectors have a clear advantage in the time and safety aspects. Another feature is that the target nucleic acid is packaged; therefore pseudotype/vectored NAT standards also serve as a control for the PCR extraction process.

The flexibility of pseudotype virus/vectored systems is another key feature that permits an expeditious response to emerging outbreaks such as Ebola. As mentioned above, the Wright laboratory has engineered pseudotype viruses for all Ebola virus species. The generation of pseudotypes to address the emergence of new Ebola strains if they appear and other outbreak viruses (e.g., Marburg, MERS) would seem to 
be not only relatively straightforward but also prudent. Also, the system lends itself to producing several thousand ampoules that are required for an International Standard whose availability is expected to last 5-10 years.

NIBSC is currently undertaking a number of collaborative studies with participating laboratories to establish WHO International Standards for Ebola antibody, NAT and antigen [11]. The studies will answer some key questions, such as how well do pseudotype virus systems perform when compared with wild-type virus in antibody neutralization assays? Are they commutable? If they are comparable then the pseudotype assay will have obvious biosafety advantages in a manner reminiscent of $\mathrm{H} 5 \mathrm{~N} 1$ influenza [12]. Are the antibody epitopes appropriately presented so that they represent relevant antigenic targets? Are packaged RNA standards appropriate surrogates for wild-type virus preparations?

The antibody standard would ideally be a pool of plasma sourced from individuals who have recovered from an Ebola infection as this should include antibodies that recognize all Ebola antigens and therefore represent an average sample that is likely to react in most assays. Such material has been difficult to collect and other sources of antibody have been included in the panel for the collaborative studies, this includes sera from vaccines and immunized transchromosomal animals producing human antibodies as described recently for

\section{References}

1 Weiss RA, Mcmichael AJ. Social and environmental risk factors in the emergence of infectious diseases. Nat. Med. 10(Suppl. 12), S70-S76 (2004).

2 Feldmann H, Jones S, Klenk HD, Schnittler HJ. Ebola virus: from discovery to vaccine. Nat. Rev. Immunol. 3(8), 677-685 (2003).

3 Mather S, Scott S, Temperton N, Wright E, King B, Daly J. Current progress with serological assays for exotic emerging/ re-emerging viruses. Future Virol. 8(8), 745-755 (2013).

4 Wool-Lewis RJ, Bates P. Characterization of Ebola virus entry by using pseudotyped viruses: identification of receptor-deficient cell lines. J. Virol. 72(4), 3155-3160 (1998).

5 Bentley EM, Mather ST, Temperton NJ. The use of pseudotypes to study viruses, virus sero-epidemiology and vaccination. Vaccine 33(26), 2955-2962 (2015). hantavirus [13]. The latter two comprise Ebola GP specific antibody and will be suitable for assays evaluating vaccine studies. A desired feature of these antibody panels is that they possess virus neutralizing activity and confirmation of neutralizing activity has been expedited by using pseudotype virus assays.

The results of these studies were made available in September 2015 and interim antibody and NAT standards were made available in October. It is unlikely that these timelines would have been achieved without the pseudotype virus/lentiviral systems to hand.

\section{Acknowledgements}

The authors would like to acknowledge E Wright (University of Westminster) and T Brooks (Public Health England) who jointly delivered the Hot Topic lecture on Ebola at the 2015 spring meeting of the Society for General Microbiology, for providing the inspiration for writing this editorial.

\section{Financial \& competing interests disclosure}

The authors have no relevant affiliations or financial involvement with any organization or entity with a financial interest in or financial conflict with the subject matter or materials discussed in the manuscript. This includes employment, consultancies, honoraria, stock ownership or options, expert testimony, grants or patents received or pending, or royalties.

No writing assistance was utilized in the production of this manuscript.
6

6 Rampling T, Ewer K, Bowyer G et al. A monovalent chimpanzee adenovirus Ebola vaccine - preliminary report. $N$. Engl. J. Med. doi:10.1056/NEJMoa1411627 (2015) (Epub ahead of print).

7 Long J, Wright E, Molesti E, Temperton N, Barclay W. Antiviral therapies against Ebola and other emerging viral diseases using existing medicines that block virus entry. F1000Res. 4, 30 (2015).

8 Mather ST, Wright E, Scott SD, Temperton NJ. Lyophilisation of influenza, rabies and Marburg lentiviral pseudotype viruses for the development and distribution of a neutralisation -assay-based diagnostic kit. J. Virol. Methods 210C, 51-58 (2014).

9 Wood JM, Major D, Heath A et al. Reproducibility of serology assays for pandemic influenza H1N1: collaborative study to evaluate a candidate WHO International Standard. Vaccine 30(2), 210-217 (2012).
10 Fryer JF, Baylis SA, Gottlieb AL et al. Development of working reference materials for clinical virology. J. Clin. Virol. 43(4), 367-371 (2008).

11 Bihamra Conference. www.biamhraconference.org

12 Alberini I, Del Tordello E, Fasolo A et al. Pseudoparticle neutralization is a reliable assay to measure immunity and crossreactivity to $\mathrm{H} 5 \mathrm{~N} 1$ influenza viruses. Vaccine 27(43), 5998-6003 (2009).

13 Hooper JW, Brocato RL, Kwilas SA et al. DNA vaccine-derived human IgG produced in transchromosomal bovines protect in lethal models of hantavirus pulmonary syndrome. Sci. Transl. Med. 6(264), 264ra162 (2014). 